\title{
Protective effects of active hexose correlated compound in a rat model of liver injury after hepatectomy
}

\author{
Richi Nakatake ${ }^{1}$, Yoshito Tanaka ${ }^{1}$, Yousuke Ueyama ${ }^{1}$, Hirokazu Miki ${ }^{1}$, Morihiko \\ Ishizaki $^{1}$, Kosuke Matsui ${ }^{1}$, Masaki Kaibori ${ }^{1}$, Tadayoshi Okumura ${ }^{1,2, *}$ \\ Mikio Nishizawa ${ }^{3}$ and Masanori Kon ${ }^{1}$
}

\footnotetext{
${ }^{1}$ Department of Surgery, Kansai Medical University, Hirakata, Osaka 573-1010, Japan; ${ }^{2}$ Research Organization of Science and Technology and ${ }^{3}$ Department of Biomedical Sciences, College of Life Sciences, Ritsumeikan University, Kusatsu, Shiga, 525-8577, Japan
}

Running title: AHCC prevents liver injury

*Corresponding Author: Tadayoshi Okumura, Ph.D., Department of Surgery, Kansai Medical University, 2-5-1 Shinmachi, Hirakata, Osaka 573-1010, Japan.

Submission Date: September 24, 2016, Accepted Date: November 24, 2016, Publication Date: November 30, 2016

Citation: Nakatake R., Tanaka Y., Ueyama Y., Miki H., Ishizaki M., Matsui K, Kaibori M, Okumura T., Nishizawa M., and Kon M. Protective effects of active hexose correlated compound in a rat model of liver injury after hepatectomy. Functional Foods in Health and Disease 2016; 6(11):702-717

\begin{abstract}
Background: Recent evidence has indicated that a functional food, active hexose correlated compound (AHCC), has liver-protective effects via suppression of inflammatory mediators, such as inducible nitric oxide synthase (iNOS) and tumor necrosis factor (TNF)- $\alpha$.
\end{abstract}

Objective: This study aimed to investigate whether AHCC has beneficial effects in a rat model of endotoxin-induced liver injury after partial hepatectomy, in addition to clarifying the mechanisms of action of AHCC.

Methods: Rats were treated with $70 \%$ of partial hepatectomy and lipopolysaccharide (PH/LPS) to induce acute liver injury. A normal diet with or without $2 \%$ AHCC was administered orally 10 days before $70 \%$ hepatectomy. Inflammatory mediators were analyzed. 
Results: AHCC improved the survival rate by $70 \%$ in PH/LPS rats. AHCC prevented an increase in serum transaminase levels, and histopathological changes and apoptosis in the liver. AHCC reduced iNOS mRNA and protein expression in the liver, resulting in inhibition of nitric oxide production. AHCC also reduced TNF- $\alpha$, cytokine-induced neutrophil chemoattractant-1, and interleukin-6 mRNA expression, but enhanced expression of interleukin-10. An electrophoretic mobility shift assay with hepatic nuclear extracts demonstrated that AHCC reduced the activation of nuclear factor (NF)- $\kappa$ B induced by PH/LPS treatment.

Conclusion: AHCC inhibits induction of inflammatory mediators, including iNOS and TNF- $\alpha$, in part through inhibition of NF- $\kappa \mathrm{B}$ activation in a rat model of liver injury. Our findings suggest that AHCC prevents postoperative liver failure after liver resection.

Keywords: active hexose correlated compound, inducible nitric oxide synthase, liver injury, nuclear factor- $\kappa \mathrm{B}$, tumor necrosis factor- $\alpha$

\section{INTRODUCTION}

Extended hepatectomy is performed for radical resection of hepatobiliary tract malignancies, but the mortality rate is not low. Hepatic failure greatly contributes to death after liver resection, and the pathophysiology involved is similar to that of sepsis [1-5]. Severe septic complications are associated with reduced phagocytic function of the reticuloendothelial system after massive hepatectomy [6]. Although two-thirds resection of the liver is not fatal in rodents, there is increased sensitivity to endotoxin in the early phase after hepatectomy. Furthermore, intravenous injection of a sublethal dose of lipopolysaccharide (LPS) postoperatively induces a high mortality [7].

Active hexose correlated compound (AHCC) is an extract from mycelia of Lentinula edodes. This is a mushroom of the basidiomycete family that is cultured in liquid medium in a large tank. AHCC is one of the functional foods that are most frequently used in cancer patients in clinical practice [8].

Animal liver injury models can be caused by various insults, such as ischemia-reperfusion, partial hepatectomy, and endotoxin shock. Using these models, we have previously reported that drugs showing liver-protective effects inhibit induction of inducible nitric oxide synthase (iNOS) and nitric oxide (NO) production [9-13]. These drugs also decrease production of various inflammatory mediators, such as tumor necrosis factor (TNF)- $\alpha$, interleukin (IL)-1 $\beta$, IL-6, and cytokine-induced neutrophil chemoattractant (CINC)-1 (human IL-8 analogue). Furthermore, by using primary cultures of rat hepatocytes, we found that AHCC, its sugar fraction, and one of its components (adenosine) inhibited nuclear factor (NF)- $\mathrm{B}$ activation, resulting in inhibition of iNOS induction and NO production [14-16]. These reports suggest that AHCC has potential effects on prevention of various liver injuries. 
However, few studies have reported that AHCC markedly enhances the survival rate in animal models of postoperative liver failure, with greater than $90 \%$ mortality. In this study, we investigated whether treatment of AHCC improves the survival rate in a rat model of endotoxin-induced liver injury after partial hepatectomy. Additionally, if the survival rate is improved, we aimed to determine whether AHCC affects expression of various inflammatory mediators, including iNOS and TNF- $\alpha$.

\section{MATERIALS AND METHODS}

Materials: The manufacturing process of AHCC is as follows: When basidiomycetes are cultured in a liquid medium, they proliferate and form globular fungal bodies rather than carpophores [17]. AHCC is produced using these bodies of mycelia of basidiomycetes and contains components of the medium that are modified by the mycelia-produced diverse enzymes. In the actual manufacturing procedure of AHCC, the mycelia of the edible shiitake are subjected to a liquid culture (15,000-L tank). After fermentation, AHCC is produced through manufacturing processes, which include separation, concentration, sterilization, and freeze-drying [18]. AHCC is certified under the "Healthy Do" system (Hokkaido Food Functionality Labelling system) in which the Hokkaido Government in Japan recognizes the conduct of scientific research on the health effects of product components. Lipopolysaccharide (LPS, Escherichia coli 0111: B4) was purchased from Sigma-Aldrich (St. Louis, MO, USA).

Experimental design: Male Sprague-Dawley rats (6 weeks old; weighing $200 \mathrm{~g}$ ) were obtained from Charles River (Tokyo, Japan). The rats were kept at $22^{\circ} \mathrm{C}$ under a $12-\mathrm{h}$ light-dark cycle and received food and water ad libitum. Rats were randomly divided into control (normal diet rat), AHCC, 70\% of partial hepatectomy and lipopolysaccharide (PH/LPS), and PH/LPS + AHCC groups. A normal diet with or without $2 \%(\mathrm{w} / \mathrm{w})$ AHCC (Amino Up Chemical Co. Ltd., Sapporo, Japan) was administered orally with free access 10 days before $70 \%$ hepatectomy, which was followed by sham operation (control and AHCC groups). Rats were anesthetized with intraperitoneal injection of pentobarbital, prior to undergoing $70 \%$ hepatectomy as reported previously $[9,10]$. After $48 \mathrm{~h}$ of surgery, LPS $(250$ $\mu \mathrm{g} / \mathrm{kg}$ ) was injected into the penile vein (PH/LPS and PH/LPS + AHCC groups). Survival was checked during $72 \mathrm{~h}$ after injection of LPS. Blood and liver samples were obtained from individual rats at 1, 3, and $6 \mathrm{~h}$ after LPS treatment.

All animal experiments were performed in accordance with the Guidelines for the Care and Use of Laboratory Animals of the National Institutes of Health. Experiments were approved by the Animal Care Committee of Kansai Medical University.

Biochemical analysis in serum: Blood samples were collected at 1, 3, and $6 \mathrm{~h}$ after LPS injection. Serum levels of aspartate transaminase (AST) and alanine transaminase (ALT) were determined using commercial kits (Wako Pure Chemical, Osaka, Japan). TNF- $\alpha$, 
CINC-1, IL-6, and IL-10 levels were measured in serum using commercial kits (R\&D Systems, Minneapolis, MN, USA). The sum of serum nitrite and nitrate $\left(\mathrm{NO}_{2}{ }^{-}\right.$and $\mathrm{NO}_{3}{ }^{-}$, respectively; stable metabolites of NO) was measured using the Griess reagent method using a commercial kit (Roche Diagnostics, Mannheim, Germany) [19].

Histopathological analysis: Excised liver specimens that were collected $6 \mathrm{~h}$ after LPS treatment were fixed in $10 \%$ formalin and embedded in paraffin. Sections $(3-5 \mu \mathrm{m})$ were cut and stained with hematoxylin and eosin. Apoptotic bodies were detected by terminal deoxynucleotidyl transferase-mediated dUTP-digoxigenin nick-end labeling (TUNEL) staining using an apoptosis detection kit (Medical and Biological Laboratories Co., Nagoya, Japan). The number of TUNEL-positive cells per square millimeter was counted. TUNEL-positive cells were quantified in a manner that was blinded to the treatment arm. All histological slides were reviewed by Dr. K. Yoshizawa (Kansai Medical University, Hirakata, Japan), the certificated pathologist by International Academy of Toxicologic Pathologists.

Western blot analysis: Frozen liver samples were homogenized in five volumes of cell solubilizing buffer (10 mM Tris-HCl, $\mathrm{pH} 7.4$; containing 1\% Triton X-100, 0.5\% Nonidet P-40, $1 \mathrm{mM}$ ethylenediaminetetraacetic acid [EDTA], $1 \mathrm{mM}$ ethyleneglycol bis [2-aminoethyl ether] tetraacetic acid, $150 \mathrm{mM} \mathrm{NaCl}, 1 \mathrm{mM}$ phenylmethylsulfonyl fluoride [PMSF], and protease inhibitor cocktail) (Roche Diagnostics) and centrifuged $(16,500 \times \mathrm{g}$ for $15 \mathrm{~min})$. The supernatant was mixed with sodium dodecyl sulfate-polyacrylamide gel electrophoresis (SDS-PAGE) sample buffer (final: $125 \mathrm{mM}$ Tris-HCl, $\mathrm{pH}$ 6.8; containing 5\% glycerol, $2 \%$ sodium dodecyl sulfate, and 1\% 2-mercaptoethanol), subjected to a $7.5 \%$ gel, and electroblotted onto a polyvinylidene-difluoride membrane (Bio-Rad, Hercules, CA, USA). Immunostaining was performed using primary antibodies against mouse iNOS (Thermo Scientific, Rockford, IL, USA) and rat $\beta$-tubulin (internal control; Clone TUB2.1; Sigma Chemical Co., St. Louis, MO, USA), followed by visualization with an enhanced chemiluminescence blotting detection reagent (GE Healthcare Biosciences Corp., Piscataway, NJ, USA). The bands corresponding to iNOS protein were quantitated by densitometry (ImageJ software) and normalized to $\beta$-tubulin.

Real-time RT-PCR: Total RNA was extracted from homogenized liver in TRIzol (Gibco BRL, USA) reagent using the guanidinium-phenol-chloroform method [20]. For strand-specific RT-PCR analysis, complementary DNAs were synthesized from total RNA with strand-specific primers, and step-down PCR was performed as previously described [21]. For iNOS (257 bp), TNF- $\alpha$ (275 bp), IL-6 (286 bp), IL-1 $\beta$ (321 bp), CINC-1 (231 bp), IL-10 (245 bp), and elongation factor-1 $\alpha$ (EF; internal control; $332 \mathrm{bp}$ ) mRNAs, an oligo (dT) primer was used for the RT reaction. The primer sets that were used for PCR are shown in Table 1. These mRNA levels were measured by real-time PCR using the Rotor-Gene Q 2plex HRM (Qiagen, Tokyo, Japan). The Rotor-Gene SYBR Green PCR Kit (Qiagen) was included 
in the reaction mixture, and the following touchdown protocol was applied: 1 cycle at $95^{\circ} \mathrm{C}$ for $5 \mathrm{~min}$, and 45 cycles at $95^{\circ} \mathrm{C}$ for $5 \mathrm{~s}$ and $60^{\circ} \mathrm{C}$ for $10 \mathrm{~s}$. Each mRNA was detected by normalizing the copy number by that of EF. The complementary DNAs for the rat iNOS mRNA were deposited in the DNA Data Bank of Japan/European Bioinformatics Institute/GenBank under the accession number AB250951.

Electrophoretic mobility shift assay: Nuclear extracts were prepared from frozen liver at $-80^{\circ} \mathrm{C}$ [22] with a minor modification. Liver sections $(0.05 \mathrm{~g})$ were homogenized with a Dounce homogenizer in $1 \mathrm{~mL}$ of buffer A (10 mM HEPES-KOH, pH 7.9; containing $10 \mathrm{mM}$ $\mathrm{KCl}, 1.5 \mathrm{mM} \mathrm{MgCl}, 1 \mathrm{mM}$ dithiothreitol, $1 \mathrm{mM} \mathrm{PMSF}$, and $5 \mu \mathrm{g} / \mathrm{mL}$ aprotinin) and allowed to swell for $15 \mathrm{~min}$. The homogenates were then centrifuged $(1100 \times \mathrm{g}$ for $5 \mathrm{~min})$. The pellet was suspended in $1 \mathrm{~mL}$ of lysis buffer (buffer A supplemented with $0.1 \%$ Triton X-100), allowed to stand for $10 \mathrm{~min}$, and centrifuged $(1100 \times \mathrm{g}$ for $10 \mathrm{~min})$. The nuclear pellet was suspended in $200 \mu \mathrm{L}$ of nuclear extraction buffer (20 mM HEPES-KOH, $\mathrm{pH} 7.9$; containing $0.42 \mathrm{M} \mathrm{NaCl}, 1.5 \mathrm{mM} \mathrm{MgCl} 2,1 \mathrm{mM}$ dithiothreitol, $1 \mathrm{mM}$ PMSF, $5 \mu \mathrm{g} / \mathrm{mL}$ aprotinin, 0.2 $\mathrm{mM}$ EDTA, and $25 \%$ [vol/vol] glycerol), incubated for $30 \mathrm{~min}$, and centrifuged $(16,500 \times \mathrm{g}$ for $20 \mathrm{~min}$ ). Binding reactions were performed by incubating the nuclear extract (5 $\mu \mathrm{g}$ protein) in reaction buffer (20 mM HEPES-KOH, pH 7.9; containing $1 \mathrm{mM}$ EDTA, $60 \mathrm{mM}$ $\mathrm{KCl}, 10 \%$ glycerol, and $1 \mu \mathrm{g}$ of poly[dI-dC]) with the probe $(40,000 \mathrm{dpm})$ for $20 \mathrm{~min}$ at room temperature. Products were electrophoresed on a $4.8 \%$ polyacrylamide gel in high ionic strength buffer, and dried gels were analyzed by autoradiography. An NF- $\kappa$ B consensus oligonucleotide (5'-AGTTGAG GGGA-CTTTCCCAGGC) from the mouse immunoglobulin light chain was purchased (Promega, Madison, WI, USA) and labeled with $\left[\gamma^{32} \mathrm{P}\right]$-adenosine triphosphate and T4 polynucleotide kinase. Protein was measured using the method of Bradford [23]. The bands corresponding to NF- $\kappa \mathrm{B}$ were quantitated by densitometry (ImageJ software).

Statistical analysis: All data are expressed as the mean \pm SE. Differences between groups and the rate of survival were identified by a two-way ANOVA and the log-rank test, respectively. $\mathrm{P}<0.05$ was considered to be statistically significant.

Table 1. Primers and nucleotide sequences

An oligo(dT) primer used for reverse transcription (RT) to synthesize complementary DNA

\begin{tabular}{|c|c|}
\hline Primer name & Nucleotide sequence \\
\hline iNOS F/R & 5' CCAACCTGCAGGTCTTCGATG 3’/5' GTCGATGCACAACTGGGTGAAC 3' \\
\hline TNF- $\boldsymbol{\alpha}$ F/R & 5' TCCCAACAAGGAGGAGAAGTTCC 3’/5' GGCAGCCTTGTCCCTTGAAGAGA 3' \\
\hline CINC-1 F/R & 5’ GCCAAGCCACAGGGGCGCCCGT 3’/5' ACTTGGGGACACCCTTTAGCATC 3' \\
\hline IL-6 F/R & 5' GAGAAAAGAGTTGTGCAATGGCA 3'/5' TGAGTCTTTTATCTCTTGTTTGAAG 3' \\
\hline HL-1 $\beta$ F/R & 5' TCTTTGAAGAAGAGCCCGTCCTC-3'/5'-GGATCCACACTCTCCAGCTGCA3' \\
\hline IL-10 & 5' GCAGGACTTTAAGGGTTACTTGG 3’/5'CCTTTGTCTTGGAGCTTATTAAA 3' \\
\hline EF F/R & 5' TCTGGTTGGAATGGTGACAACATGC 3'/5' CCAGGAAGAGCTTCACTCAAAGCTT 3' \\
\hline
\end{tabular}


(cDNA) to mRNA. iNOS, inducible nitric oxide synthase; TNF- $\alpha$, tumor necrosis factor- $\alpha$; CINC-1, cytokine-induced neutrophil chemoattractant-1; IL-6, interleukin-6; IL-1 $\beta$, interleukin-1 $\beta ; \mathrm{EF}$, elongation factor-1 $\alpha . \mathrm{F} / \mathrm{R}$, forward/reverse.

\section{RESULTS}

\section{AHCC enhanced the survival of rats with liver injury}

More than $90 \%$ of rats died within $72 \mathrm{~h}$ after treatment of $70 \%$ hepatectomy and LPS (PH/LPS). Pretreatment of normal diet with $2 \%$ AHCC in PH/LPS-treated rats enhanced the survival rate by $70 \%$ (Fig. 1). Serum levels of transaminases (AST and ALT), which are the markers of hepatocellular damage, were reduced with treatment of AHCC (Fig. 2).

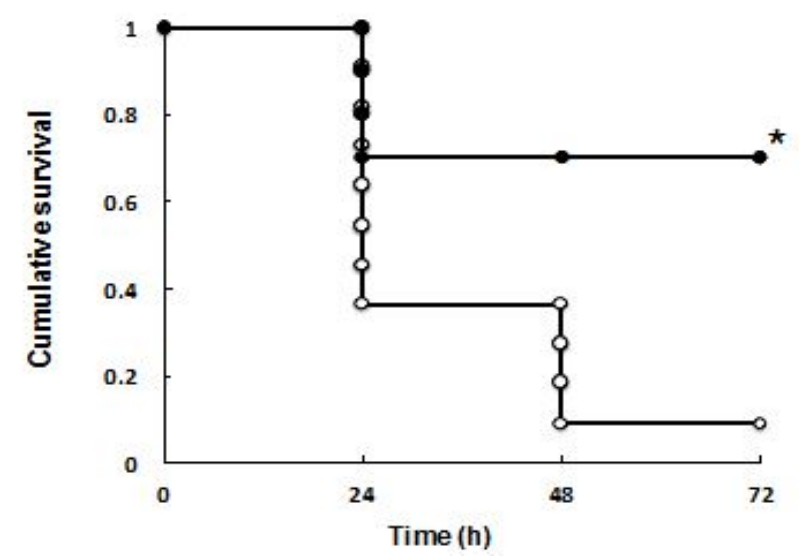

Fig. 1. Effect of AHCC on survival of rats. Rats were treated with $70 \%$ partial hepatectomy $(\mathrm{PH})$ and lipopolysaccharide (LPS). A normal diet with or without $2 \%$ AHCC was administered orally 10 days before PH treatment. Data represent the percentage of survival at the indicated times after LPS. PH/LPS rats: open circles, $\mathrm{n}=10 ; \mathrm{PH} / \mathrm{LPS}+\mathrm{AHCC}$ rats: filled circles, $\mathrm{n}=10 . * \mathrm{P}<0.05$ versus $\mathrm{PH} / \mathrm{LPS}$ rats.
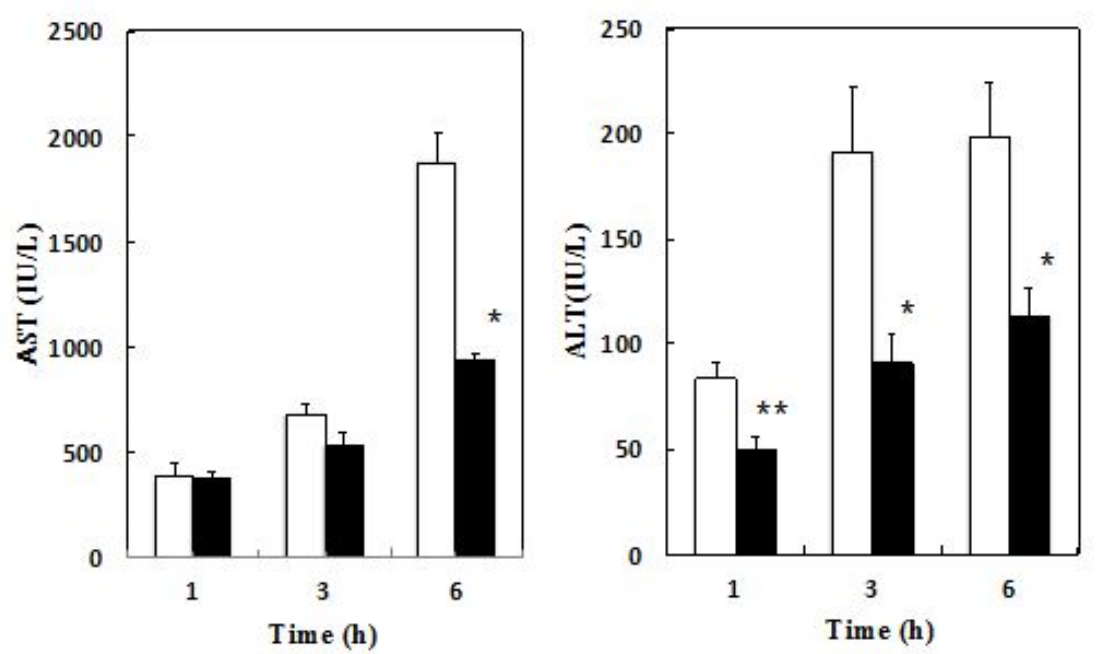

Fig. 2. Effect of AHCC on serum AST and ALT levels. Rats were treated with $70 \%$ partial hepatectomy (PH) and lipopolysaccharide (LPS). A normal diet with or without $2 \%$ AHCC was administered orally 10 days before PH treatment. Blood samples were collected at the indicated times after LPS. Data represent the mean $\pm \mathrm{SE}$ ( $\mathrm{n}=5-6$ rats per time point per group). PH/LPS (open), PH/LPS + AHCC (closed). ${ }^{*} \mathrm{P}<0.05$ and **P $<0.01$ versus $\mathrm{PH} / \mathrm{LPS}$ rats. 


\section{AHCC reduced the appearance of pathological changes}

Histological alteration of the liver in PH/LPS rats was characterized by inflammatory cell infiltration, hemorrhagic changes, and focal necrosis in the midzone and periportal regions. Focal hepatocyte necrosis with hemorrhagic changes, ballooning degeneration, and inflammatory cell infiltration were observed in the liver $6 \mathrm{~h}$ after PH/LPS (Fig. 3A), and AHCC prevented these pathological changes (Fig. 3B). PH/LPS treatment dramatically increased apoptosis of hepatocytes in the liver (Fig. 3C). AHCC markedly reduced apoptosis in hepatocytes and decreased the apoptotic index of the liver (Fig. 3D and 3E). In rats that were treated with AHCC alone (without PH/LPS), the liver showed normal hepatic histology (data not shown).

\section{PH/LPS}

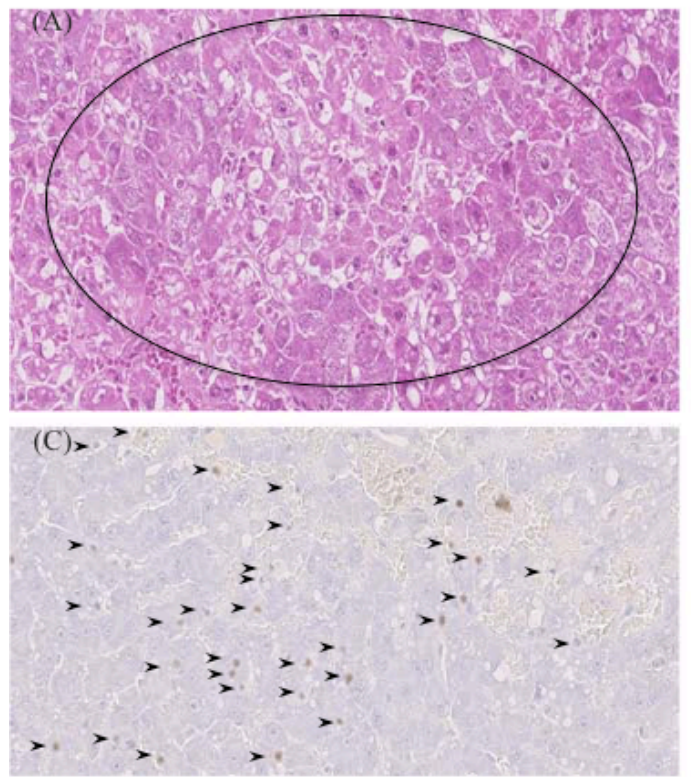

PH/LPS + AHCC

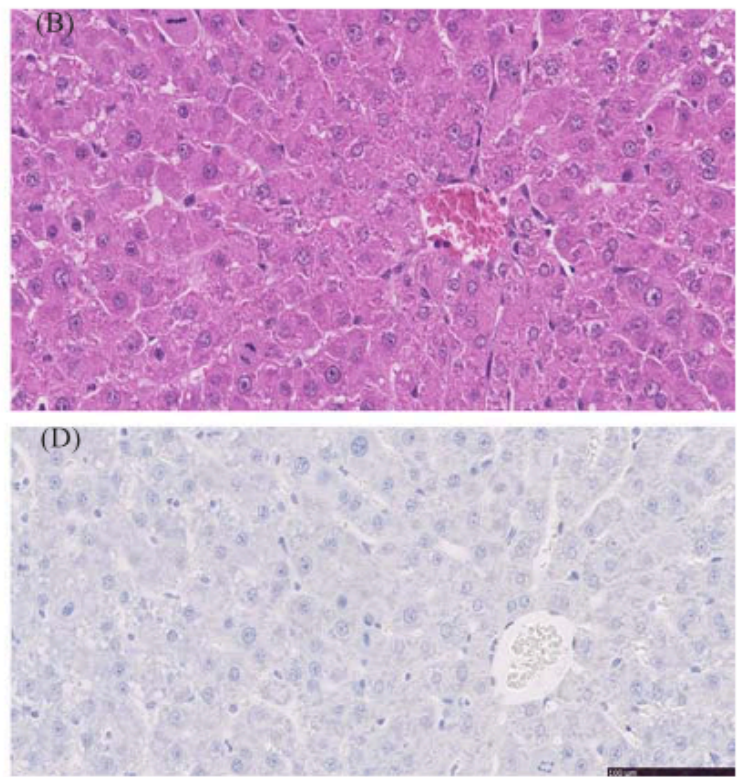

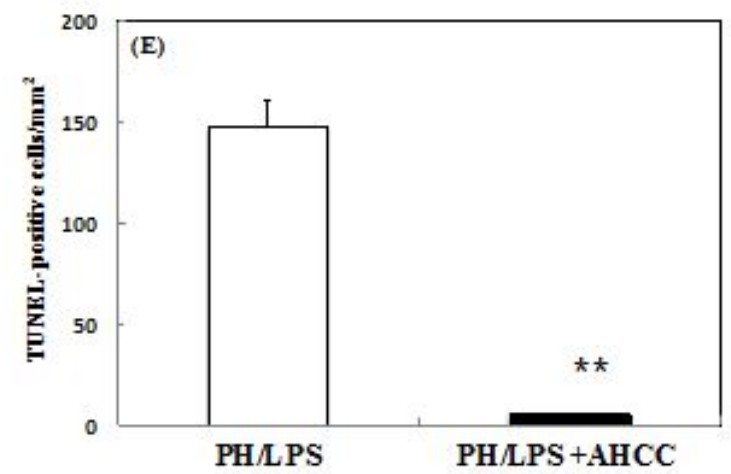

Fig. 3. Effect of AHCC on pathological changes in the liver. Rats were treated with $70 \%$ partial hepatectomy (PH) and lipopolysaccharide (LPS). A normal diet with or without $2 \%$ AHCC was administered orally before PH treatment. (A and B): histological appearance of the liver after PH/LPS treatment was evaluated. Liver samples of (A) PH/LPS and (B) PH/LPS + AHCC were obtained $6 \mathrm{~h}$ after PH/LPS treatment and stained with hematoxylin and eosin (magnification x 200, allow heads indicate apoptotic cells). Circled areas indicate the salient features (focal necrosis with inflammatory cell infiltration and massive hemorrhagic change). (C, D, and E): the effect of AHCC on apoptosis in the liver was evaluated. Liver sections of (C) PH/LPS and (D) PH/LPS + AHCC that were obtained $6 \mathrm{~h}$ after treatment were stained with TUNEL (magnification $\times 200$ ). Data represent the mean $\pm \mathrm{SE}$ ( $\mathrm{n}=5-6$ per group). ${ }^{*} \mathrm{P}<0.05$ versus $\mathrm{PH} / \mathrm{LPS}$ rats. The number of TUNEL-positive cells per square millimeter was counted (E). 


\section{AHCC affected the expression of iNOS and cytokines in the liver}

During endotoxemia, excess production of NO caused by iNOS has a crucial role in hepatic dysfunction. PH/LPS treatment increased iNOS mRNA levels and AHCC inhibited its expression in the liver (Fig. 4A). There was a negligible expression of iNOS mRNA in the normal and AHCC alone (without PH/LPS) rat groups and there was no significant difference between the two groups (data not shown). Western blotting showed that PH/LPS treatment increased iNOS protein expression at $3 \mathrm{~h}$ and AHCC reduced its expression (Fig. 4B). In support of these observations, serum levels of nitrite and nitrate, which are stable metabolites of NO, increased after PH/LPS treatment at $6 \mathrm{~h}$. AHCC inhibited this increase in nitrite and nitrate levels (Fig. 4C).

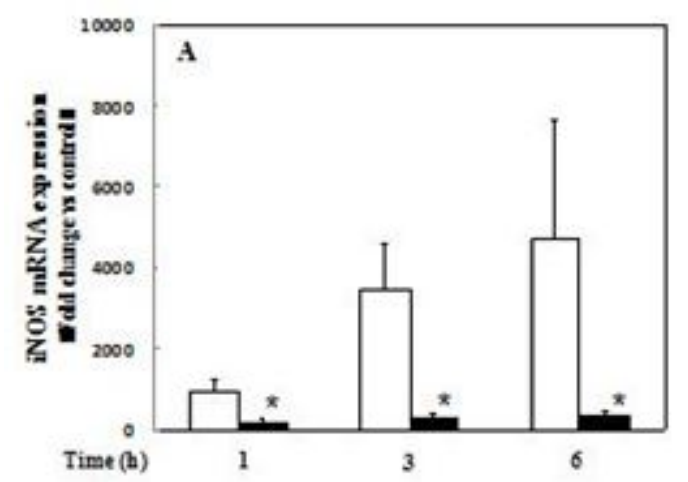

B

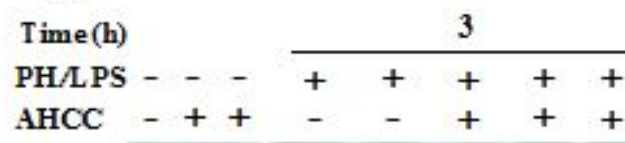

iNOS protein

$(130 \mathrm{kD})$

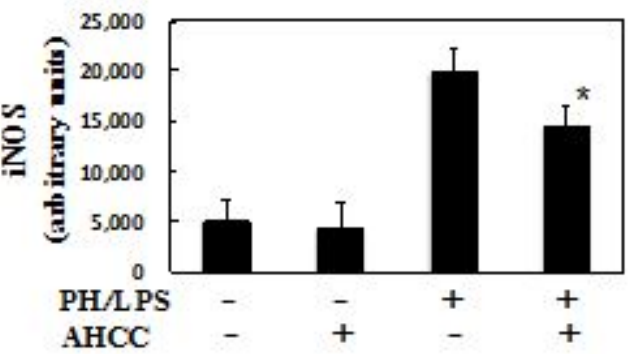

C

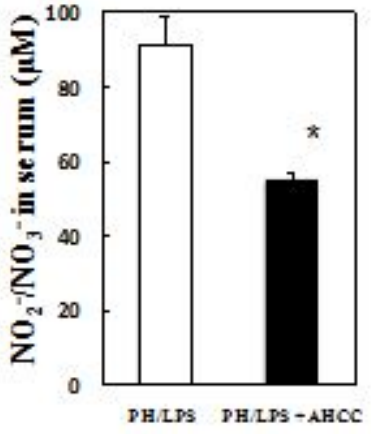

Fig. 4. Effect of AHCC on induction of iNOS and nitric oxide production in the liver. Rats were treated with $70 \%$ partial hepatectomy $(\mathrm{PH})$ and lipopolysaccharide (LPS). A normal diet with or without 2\% AHCC was administered orally before PH treatment. (A) Total RNA was obtained at the indicated times after LPS treatment and was analyzed by strand-specific quantitative RT-PCR to detect iNOS. Data were calculated as the fold change versus normal diet rats (control group). Data represent the mean \pm SE $(n=5-6$ rats per time point per group). (B) For western blotting, liver samples were obtained before or at $3 \mathrm{~h}$ after LPS treatment. The cell lysate (100 mg protein) was subjected to SDS-PAGE in a $7.5 \%$ gel and immunoblotted with anti-iNOS or anti- $\beta$-tubulin antibodies. Representative results of three independent experiments are shown. The bands corresponding to iNOS protein were quantitated by densitometry ( $\mathrm{n}=3$ experiments). (C): blood samples were collected at $6 \mathrm{~h}$ after LPS treatment and the levels of $\mathrm{NO}_{2}{ }^{-}$and $\mathrm{NO}_{3}{ }^{-}$(metabolites of nitric oxide) were measured. Data represent the mean $\pm \mathrm{SE}$ ( $\mathrm{n}=5-6$ rats per time point per group). PH/LPS (open), PH/LPS + AHCC (closed) at $(\mathrm{A})$ and $(\mathrm{C}) .{ }^{*} \mathrm{P}<0.05$ versus $\mathrm{PH} / \mathrm{LPS}$ rats. 
Real-time RT-PCR analysis showed that PH/LPS treatment increased mRNA expression of TNF- $\alpha$, CINC-1, IL-6, and IL-1 $\beta$. AHCC decreased mRNA expression of TNF- $\alpha$, CINC-1, and IL-6 (Fig. 5A, 5B and 5C, respectively), but had no effect on IL-1 $\beta$ mRNA expression (Fig. 5D). In contrast, AHCC increased anti-inflammatory IL-10 mRNA expression (Fig. 5E), whereas PH/LPS treatment had no effect on IL-10 mRNA expression. There was a negligible expression of TNF- $\alpha$, CINC-1, IL-6, IL-1 $\beta$ and IL-10 mRNA in the normal and AHCC alone (without PH/LPS) rat groups (data not shown). In serum analysis, AHCC increased IL-10 levels (Fig. 6D), but there were no significant differences in other serum cytokines between the two groups (Fig. 6A, 6B, and 6C).
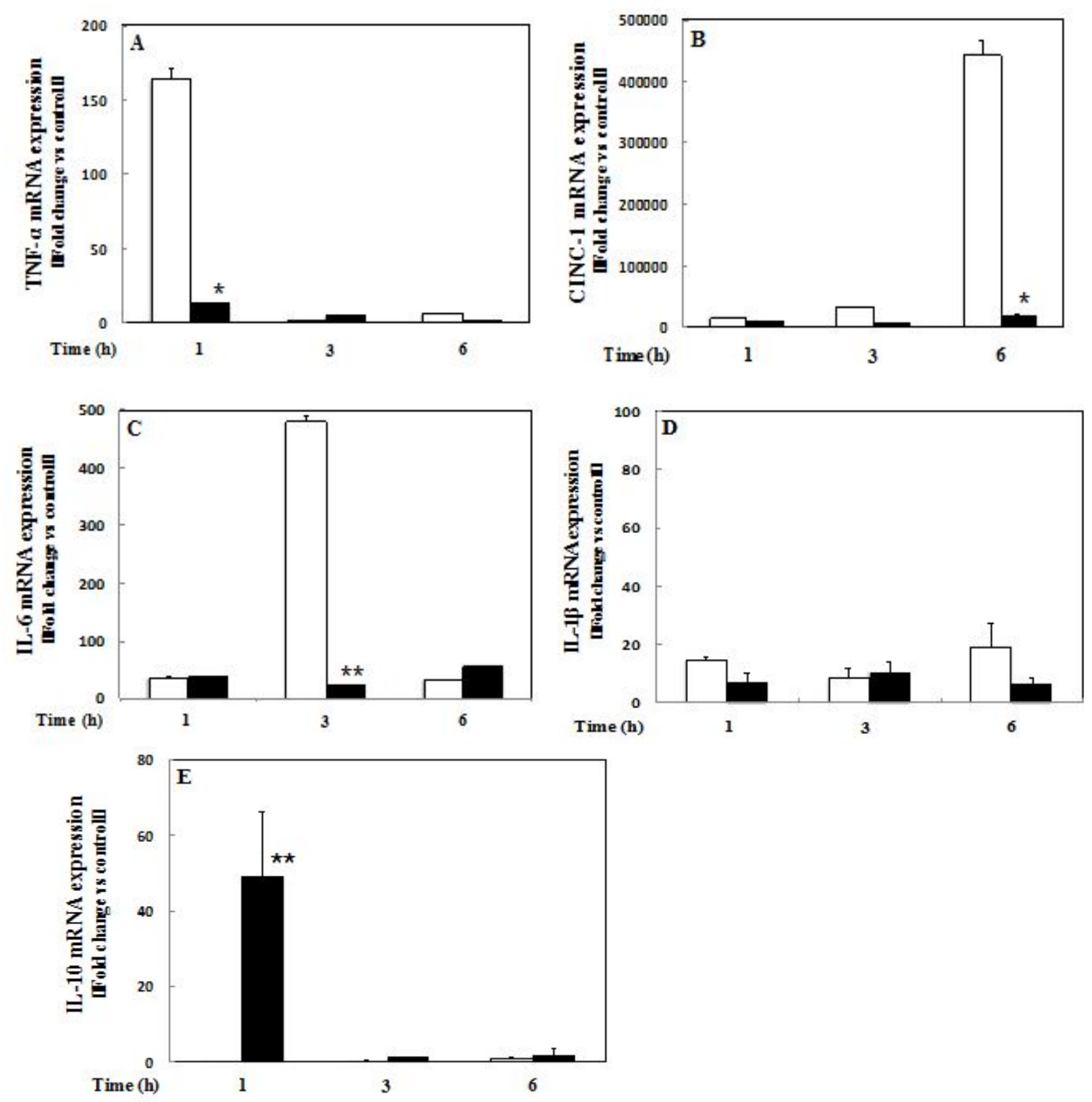

Fig. 5. Effect of AHCC on mRNA expression of cytokines in the liver. Rats were treated with $70 \%$ partial hepatectomy (PH) and LPS. A normal diet with or without $2 \%$ AHCC was administered orally before PH treatment. Total RNA was extracted from the liver at the indicated times after LPS treatment and was analyzed by strand-specific real-time RT-PCR to detect (A) TNF- $\alpha$, (B) CINC-1, (C) IL-6, (D) IL-1 $\beta$, and (E) IL-10. Data were calculated as the fold change versus normal diet rats (control group). Data represent the mean \pm $\mathrm{SE}(\mathrm{n}=5-6$ rats per time point per group). $\mathrm{PH} / \mathrm{LPS}$ (open), $\mathrm{PH} / \mathrm{LPS}+$ AHCC (closed). $* \mathrm{P}<0.05$ and $* * \mathrm{P}<0.01$ versus $\mathrm{PH} / \mathrm{LPS}$ rats. 


\section{AHCC inhibited the activation of $\mathrm{NF}-\mathrm{kB}$ in the liver}

Recent evidence has indicated that NF-kB is involved in the transcriptional activation of a variety of inflammatory genes, such as iNOS, TNF- $\alpha$, and CINC-1. We examined the effect of AHCC on NF-kB activation (its nuclear translocation and DNA binding) in the liver. The electrophoretic mobility shift assay with hepatic nuclear extracts showed that PH/LPS treatment stimulated activation of NF-kB at $3 \mathrm{~h}$ and AHCC reduced the levels of NF-kB activation (Fig. 7).
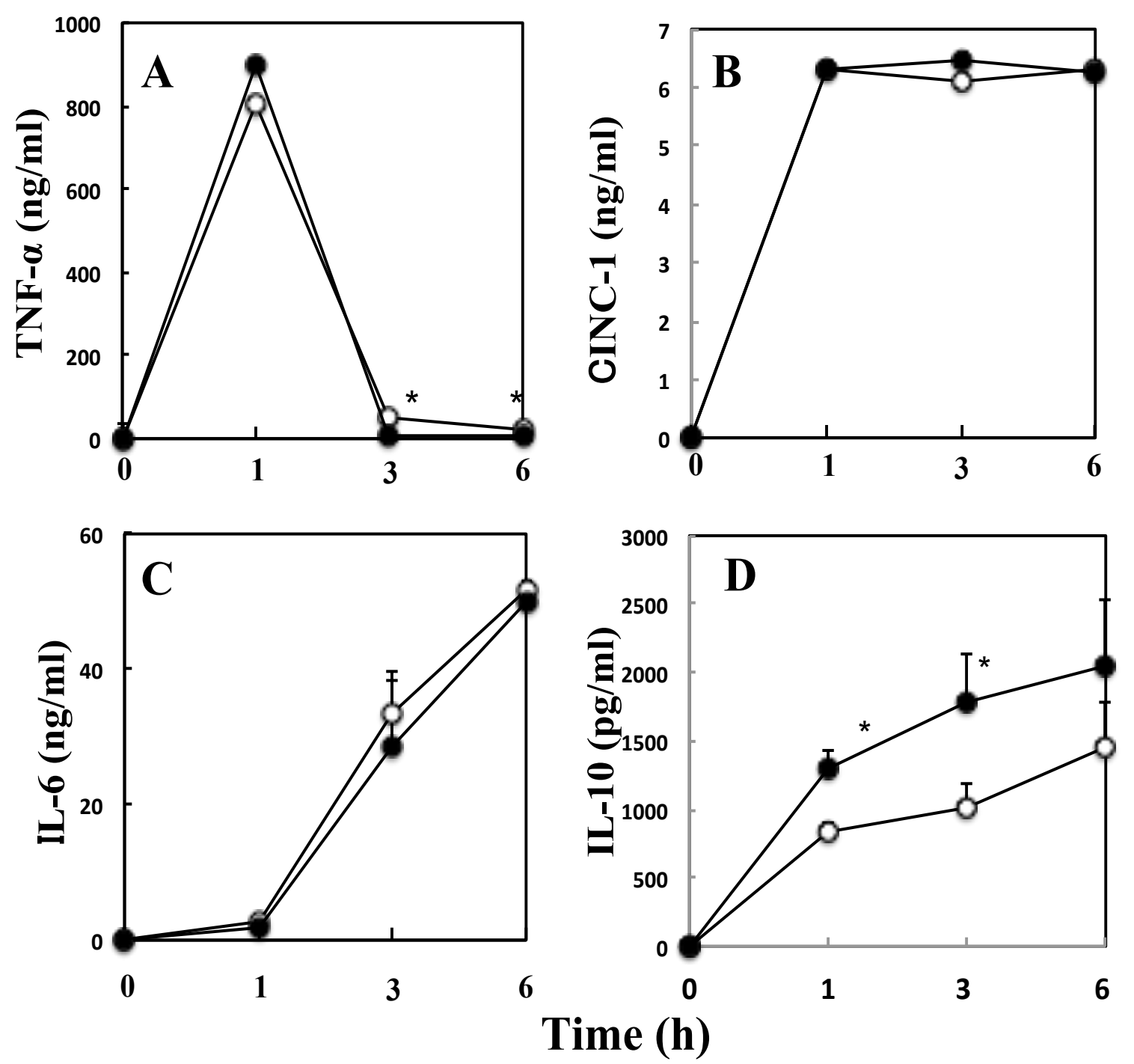

Fig. 6. Effect of AHCC on serum cytokine levels. Rats were treated with $70 \%$ partial hepatectomy (PH) and LPS. A normal diet with or without $2 \%$ AHCC was administered orally before PH treatment. Blood samples were collected at the indicated times after LPS treatment. Serum levels of (A) TNF- $\alpha$, (B) CINC-1, (C) IL-6, and (D) IL-10 were measured. Data represent the mean \pm SE ( $\mathrm{n}=$ 5-6 rats per time point per group). PH/LPS rats: open circles; PH/LPS + AHCC rats: filled circles. ${ }^{*} \mathrm{P}<$ 0.05 versus $\mathrm{PH} / \mathrm{LPS}$ rats. 


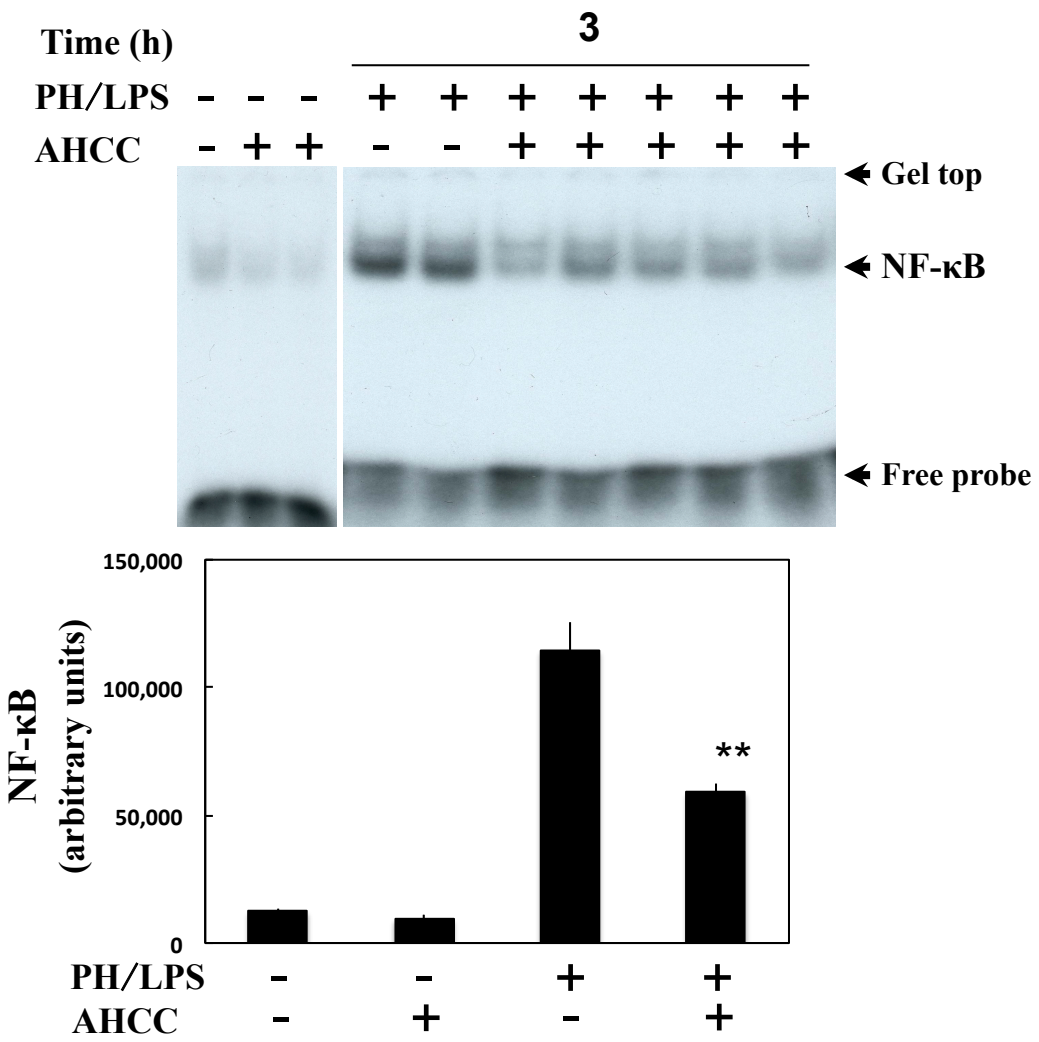

Fig. 7. Effect of AHCC on activation of NF-kB in the liver. Rats were treated with $70 \%$ hepatectomy $(\mathrm{PH})$ and LPS. A normal diet with or without $2 \%$ AHCC was administered orally before $\mathrm{PH}$ treatment. Nuclear extracts ( $4 \mu \mathrm{g}$ of protein) were prepared from the liver before or at $3 \mathrm{~h}$ after LPS treatment, and NF-kB was analyzed by an electrophoretic mobility shift assay (upper panel). Representative results of three independent experiments are shown. The bands corresponding to NF-kB were quantitated by densitometry (lower panel; $\mathrm{n}=3$ experiments). PH/LPS (open), PH/LPS + AHCC (closed). $* * \mathrm{P}<0.01$ versus PH/LPS rats.

\section{DISCUSSION}

In our preliminary study, a normal diet with $1 \%$ AHCC demonstrated a tendency to increase the survival rate of rats, but this was not significant (Tanaka and Nakatake, unpublished observation). In the current study, we found that oral intake of a normal diet with $2 \%$ AHCC before treatment of PH/LPS attenuated the increase in AST and ALT levels (Fig. 2), and reduced the appearance of pathological changes, including apoptosis (Fig. 3). This led to marked improvement in the survival rate of rats (Fig. 1).

AHCC is abundant in the $\alpha$-glucan form of carbohydrates. The presence of $\alpha$-1,4-glucan, in which the hydroxyl groups of C-2 and/or C-3 position are partially acylated, is particularly reported and is considered to be one of the active ingredients [18]. Based on a survey carried out by the Ministry of Health, Labor and Welfare research group in Japan, AHCC is the second most commonly used health food, followed by Agaricus, which is a fungus, among cancer patients in Japan [8]. AHCC is useful for treating various diseases, including hepatitis and cancer, as a complementary and alternative medicine [24]. We reported that AHCC improved the prognosis of patients with hepatocellular carcinoma postoperatively (a prospective cohort study) [25]. AHCC is effective for treating infectious and inflammatory 
diseases, and is a cancer therapy [26-32]. The safety of AHCC has been supported by basic studies, including its interaction with drugs [33-35]. Results of a randomized, controlled trial on healthy volunteers strongly supported the safety of AHCC as a supplement in clinical practice [36].

In this study, AHCC inhibited the expression of iNOS mRNA and its protein, leading to inhibition of NO production (Fig. 4A, 4B, and 4C). AHCC decreased mRNA expression of TNF- $\alpha$, CINC-1, and IL-6 in the liver (Fig. 5A, 5B, and 5C). However, we did not find a reduction in serum TNF- $\alpha$, CINC-1, and IL- 6 levels. This finding was unexpected and the reasons currently remain unclear. AHCC enhanced IL-10 levels in the liver and serum (Figs. $5 \mathrm{E}$ and 6D). Accordingly, part of the protective effects of AHCC in our rat model of endotoxin-induced acute liver injury probably resulted from attenuation of iNOS, TNF- $\alpha$, CINC-1, and IL-6, and enhancement of IL-10.

One of the earliest events after interaction of LPS with toll-like receptor 4 is activation of transcription factor NF- $\mathrm{\kappa B}$ in cells, with its translocation from the cytoplasm to the nucleus and DNA binding [37]. Once activated, NF- $\mathrm{B}$ induces transcriptional upregulation of various inflammatory mediators, such as iNOS, TNF- $\alpha$, and CINC-1 [38-40]. The electrophoretic mobility shift assay with nuclear extracts from the liver showed that AHCC decreased activation of NF- $\kappa \mathrm{B}$ induced by PH/LPS treatment (Fig. 7).

\section{CONCLUSION}

AHCC decreased mRNA expression of inflammatory mediators, such as iNOS and TNF- $\alpha$, in PH/LPS-treated rats. This was achieved in part through inhibition of NF-kB activation, resulting in prevention of liver injury. AHCC may have therapeutic potential for use in prevention of postoperative liver failure after liver resection.

Abbreviations: AHCC, active hexose correlated compound; PH/LPS, 70\% of partial hepatectomy and lipopolysaccharide; iNOS, inducible nitric oxide synthase; TNF- $\alpha$, tumor necrosis factor-alpha; NF-кB, nuclear factor-kappa B. IL, interleukin; CINC-1, cytokine-induced neutrophil chemoattractant-1.

Disclosure of potential conflicts of interest: The authors declare that they have no conflicts of interest.

Author's contributions: RN participated in the design of the study, data collection, statistical analysis, and drafting of the manuscript. YT and YU participated in supervision and provided advice when the manuscript was being drafted. HM assisted in data collection and analysis. MI, KM, and MK assisted in the design of the study. TO, MN and MKon provided advice regarding protocol development for the study and assisted in the design of the study.

Acknowledgments: This work was supported in part by a Grant-in-Aid for Scientific 
Research from the Ministry of Education, Science, Culture and Sports of Japan, and by grants from the Science Research Promotion Fund of the Japan Private School Promotion Foundation. The authors wish to thank Dr. K. Yoshizawa for providing valuable comments in histological analysis.

\section{REFERNCES}

1. Chen M, Hwang T, Jeng L, Jan Y, Wang C: Influence of age on results of resection of hepatocellular carcinoma. Eur J Surg 1991, 157: 591-593.

2. Iwatsuki S, Starzl TE: Personal experience with 411 hepatic resections. Ann Surg 1988, 208: 421- 434.

3. Lind DS, Parker GA, Horsley JS 3rd, Kornstein MJ, Neifeld JP, Bear HD, et al.: Formal hepatic resection of colorectal liver metastases: ploidy and prognosis. Ann Surg 1992, 215: 677-684.

4. Al-Hadeedi S, Choi TK, Wong J: Extended hepatectomy for hepatocellular carcinoma. Br J Surg 1990, 77: 1247-1250.

5. Boermeester MA, Straatsburg IH, Houdijk AP, Meyer C, Frederiks WM, Wesdorp RI, et al.: Endotoxin and interleukin-1 related hepatic inflammatory response promotes liver failure after partial hepatectomy. Hepatology 1995, 22: 1499-1506.

6. Arii S, Shibagaki M, Takahashi Y, Taki Y, Yokoo N, Jikko A, et al. :Changes in the reticuloendothelial phagocytic function after partial hepatectomy. J Lab Clin Med 1985,105: 668-672.

7. Mochida S, Ogata I, Hirata K, Ohta Y, Yamada S, Fujiwara K: Provocation of massive hepatic necrosis by endotoxin after partial hepatectomy in rats. Gastroenterology 1990, 99: 771-777.

8. Hyodo I, Amano N, Eguchi K, Narabayashi M, Imanishi J, Hirai M, et al.: Nationwide survey on complementary and alternative medicine in cancer patients in Japan. J Clin Oncol 2005, 23: 2645-2654.

9. Tsuchiya H, Kaibori M, Yanagida H, Yokoigawa N, Kwon A-H, Okumura T, Kamiyama Y: Pirfenidone prevents endotoxin-induced liver injury after partial hepatectomy in rats. J Hepatol 2004, 40: 94-101.

10. Tsuji K, Kwon A-H, Yoshida H, Qiu Z, Kaibori M, Okumura T, Kamiyama Y: Free radical scavenger (edaravone) prevents endotoxin-induced liver injury after partial hepatectomy in rats. J Hepatol 2005, 42: 94-101.

11. Tanaka T, Uchida Y, Kaibori M, Hijikawa T, Ishizaki M, Yamada M, Matsui K, Ozaki T, Tokuhara K, Kamiyama Y, Nishizawa M, Ito S, Okumura $\mathrm{T}: \mathrm{Na}^{+} / \mathrm{H}^{+}$exchanger inhibitor, FR183998, has protective effect in lethal acute liver failure and prevents iNOS induction in rats. J Hepatol 2008, 48: 289-299.

12. Hijikawa T, Kaibori M, Uchida Y, Yamada M, Matsui K, Ozaki T, Kamiyama Y, Nishizawa M, Okumura T: Insulin-like growth factor-I prevents liver injury through the inhibition of TNF- $\alpha$ and iNOS induction in D-galactosamine and 
lipopolysaccharide-treated rats. Shock 2008, 29: 740-747.

13. Ishizaki M, Kaibori M, Uchida Y, Hijikawa T, Tanaka H, Ozaki T, Tokuhara K, Matsui K, Kwon A-H, Kamiyama Y, Nishizawa M, Okumura T: Protective effect of FR183998, a Na $/ \mathrm{H}^{+}$exchanger inhibitor, and its inhibition of iNOS induction in hepatic ischemia-reperfusion injury in rats. Shock 2008, 30: 311-317.

14. Matsui K, Kawaguchi Y, Ozaki T, Tokuhara K, Tanaka H, Kaibori M, Matsui Y, Kamiyama Y, Wakame K, Miura T, Nishizawa M, Okumura T: Effect of active hexose correlated compound on the production of nitric oxide in hepatocytes. JPEN J Parenter Enteral Nutr 2007, 31: 373-381.

15. Matsui K, Ozaki T, Oishi M, Tanaka Y, Kaibori M, Nishizawa M, Okumura T, Kwon A-H: Active hexose correlated compound inhibits the expression of proinflammatory biomarker iNOS in hepatocytes. Eur Surg Res 201, 47: 274-283.

16. Tanaka Y, Ohashi S, Ohtsuki A, Kiyono T, Park EY, Nakamura Y, Sato K, Oishi M, Miki H, Tokuhara K, Matsui K, Kaibori M, Nishizawa M, Okumura T, Kwon A-H: Adenosine, a hepato-protective component in active hexose correlated compound: its identification and iNOS suppression mechanism. Nitric Oxide 2014, 40: 75-86.

17. Furukawa H: 1992. In Science of Mushroom, 1st ed. 72. Japan: Kyoritsu Publications.

18. Miura T, Kitadate K, Nishioka H, Wakame K: "Basic and clinical studies on active hexose correlated compound". In Biotechnology in Functional Foods and Nutraceuticals: 51-59, 2010.

19. Green LC, Wagner DA, Glogowski J, Skipper PL, Wishnok JS, Tannenbaum SR: Analysis of nitrate, nitrite and $(15 \mathrm{~N})$ nitrate in biological fluids. Anal Biochem 1982, 126(1): 131-138.

20. Chomczynski P, Sacchi N: Single-step method of RNA isolation by acid guanidinium thiocyanate-phenol-chloroform extraction. Anal Biochem 1987, 162: 156-159.

21. Nishizawa $M$, Nakajima $T$, Yasuda $K$, et al.: Close kinship of human 20alpha-hydroxysteroid dehydrogenase gene with three aldo-keto reductase genes. Genes Cells 2000, 5: 111-125.

22. Essani N, McGuire G, Manning A, Jaeschke H: Endotoxin-induced activation of the nuclear transcription factor $\mathrm{B}$ and expression of E-selectin messenger RNA in hepatocytes, Kupffer cells, and endothelial cells in vivo. J Immunol 1996, 156: 2956-63.

23. Bradford MM: A rapid and sensitive method for the quantitation of microgram quantities of protein utilizing the principle of protein-dye binding. Anal Biochem 1976; 72: 248-254.

24. Parida DK, Wakame K, Nomura T: Integrating complementary and alternative medicine in form of active hexose correlated compound (AHCC) in the management of head \& neck cancer patients. Int J Clin Med 2011, 2: 588-592.

25. Matsui Y, Uhara J, Satoi S, Kaibori M, Yamada H, et al.: Improved prognosis of postoperative hepatocellular carcinoma patients when treated with functional foods: a 
prospective cohort study. J Hepatol 2002, 37: 78-86.

26. Thaiudom S, Piyaniran W, Chutaputthi A: A study of the efficacy of active hexose correlated compound (AHCC) in the treatment of chronic hepatitis $\mathrm{C}$ patients at Phramongkutklao Hospital. Med News Thailand 2010, 13-16.

27. Kim JH, Im JA: Effect of active hexose correlated compound (AHCC) in alcoholic fatty liver patients. J Nutr Sci Vitaminol 2014, 60: 340-346.

28. Roman BE, Beli E, Duriancik DM, Gardner EM: Short-term supplementation with active hexose correlated compound improves the antibody response to influenza B vaccine. Nutrition Res 2013, 33: 12-17.

29. Ito T, Urushima H, Sakaue M, Yukawa S, and Honda H.: Reduction of adverse effects by a mushroom product, active hexose correlated compound (AHCC) in patients with advanced cancer during chemotherapy - the significance of the levels of HHV-6 DNA in saliva as a surrogate biomarker during chemotherapy. Nutrition Cancer 2014, 370: 377-382.

30. Hangai S, Iwase S, Kawaguchi T, Kogure Y, Miyaji T, et al.: Effect of active hexose-correlated compound in women receiving adjuvant chemotherapty for breast cancer: a retrospective study. J Altern Complement Med 2013, 19: 905-910.

31. Cowawintaweewat S, Manoromana S, Sriplung H, Khuhaprema T, Tongtawe P, et al.: Prognostic improvement of patients with advanced liver cancer after active hexose correlated compound (AHCC) treatment. Asian Pac J Allergy Immunol 2006, 24 : $33-45$.

32. Ishizuka R, Fujii H, Miura T, Fukuchi Y, Tajima K: Personalized cancer therapy for stage IV non-small cell lung cancer: combined use of active hexose correlated compound and genistein concentrated polysaccharide. Pers Med Univ 2012, 1: 39-44.

33. Fujii H, Nishioka H, Simon RR, Kaur R, Lynch B et al.: Genotoxicity and subchronic toxicity evaluation of active hexose correlated compound (AHCC). Regul Toxicol Pharmacol 2011, 59: 237-250.

34. Mach CM, Fujii H, Wakame K, Smith J: Evaluation of active hexose correlated compound hepatic metabolism and potential for drug interactions with chemotherapy agents. J Soc Integr Oncol 2008, 6: 105-109.

35. Spierings ELH, Fujii H, Sub B, Walshe T: A phase I study of the safety of the nutritional supplement, active hexose correlated compound, AHCC, in healthy volunteers. J Nutr Sci Vitaminol 2007, 53: 536-539.

36. Terakawa N, Matsui Y, Satoi S, Yanagimoto H, Takahashi K, et al.: Immunological effect of active hexose correlated compound (AHCC) in healthy volunteers: a double-blind, placebo-controlled trial. Nutrition Cancer 2008, 60: 643-651.

37. Bellezzo J, Britton R, Bacon B, Fox E: LPS-mediated NF-kappaB activation in rat Kupffer cells can be induced independently of CD14. Am J Physiol 1996, 270 (6 Pt 1): G956.

38. Siebenlist U, Franzoso G, Brown K: Structure, regulation and function of NF-kappa B. 
Annu Rev Cell Biol 1994, 10: 405.

39. Su G: Lipopolysaccharides in liver injury: molecular mechanisms of Kupffer cell activation. Am J Physiol Gastrointest Liver Physiol 2002, 283: G256.

40. Blackwell T, Holden E, Blackwell T, DeLarco J, Christman J: Cytokine-induced neutrophil chemoattractant mediates neutrophilic alveolitis in rats: association with nuclear factor kappa B activation. Am J Respir Cell Mol Biol 1994, 11: 464. 